\title{
Four-dimensional Magnetic Resonance Imaging with 3D Radial Sampling and Self-gating based K-space Sorting: Early Clinical Experience on Pancreatic Cancer Patients
}

\author{
Wensha Yang, $\mathrm{PhD}^{1,{ }^{*}}$, Zhaoyang Fan, $\mathrm{PhD}^{2}$, Richard Tuli, MD, $\mathrm{PhD}^{1}$, Zixin Deng, $\mathrm{MS}^{2}$, \\ Jianing Pang, $\mathrm{PhD}^{2}$, Ashley Wachsman, MD³ , Robert Reznik, MD¹, Howard Sandler, MD, \\ MS ${ }^{1}$, Debiao Li, PhD², and Benedick A. Fraass, PhD $^{1}$ \\ ${ }^{1}$ Department of Radiation Oncology, Cedars Sinai Medical Center, Los Angeles, CA \\ 2Biomedical Imaging Research Institute, Department of Biomedical Sciences, Cedars Sinai \\ Medical Center, Los Angeles, CA \\ ${ }^{3}$ Department of Imaging, Cedars Sinai Medical Center, Los Angeles, CA
}

\begin{abstract}
Purpose-Dynamic magnetic resonance imaging (MRI) has been used to characterize internal organ motion but real time acquisition is typically limited to 2 dimensions. Methods have been developed to reconstruct four dimensional MRI (4D-MRI) based on time-stamped 2D images or 2D K-space data. These methods suffer from anisotropic resolution and rebinning artifacts. We applied a novel self-gating K-space sorted 4D-MRI (SG-KS-4D-MRI) method to overcome these limitations and to monitor pancreatic tumor motion.
\end{abstract}

Methods and Material-Ten patients were imaged using 4D-CT, cine 2D-MRI and the SGKS-4D-MRI, which is a spoiled gradient recalled echo (GRE) sequence with 3D radial-sampling $\mathrm{K}$-space projections and $1 \mathrm{D}$ projection-based self-gating. Tumor volumes were defined on all phases in both 4D-MRI and 4D-CT and then compared.

Results-An isotropic resolution of $1.56 \mathrm{~mm}$ was achieved in the SG-KS-4D-MRI images, which showed superior soft tissue contrast to 4D-CT and appeared to be free of visible rebinning artifacts. The tumor motion trajectory cross-correlations between SG-KS-4D-MRI and cine 2DMRI in SI, AP and ML directions were $0.93 \pm 0.03,0.83 \pm 0.10$ and $0.74 \pm 0.18$, respectively. The tumor motion trajectories cross-correlations between SG-KS-4D-MRI and 4D-CT in SI, AP and ML directions were $0.91 \pm 0.06,0.72 \pm 0.16$ and $0.44 \pm 0.24$, respectively. The average standard deviation of GTV volume (GTV_o) calculated from the ten breathing phases were $0.81 \mathrm{cc}$ and $1.02 \mathrm{cc}$ for SG-KS-4D-MRI and 4D-CT ( $\mathrm{p}=0.012)$.

\footnotetext{
*Corresponding author: Wensha Yang, PhD, Department of Radiation Oncology, Cedars Sinai Medical Center, 8700 Beverly Blvd., Los Angeles, CA 90048, wensha.yang@cshs.org.

Conflicts of interest: none

Publisher's Disclaimer: This is a PDF file of an unedited manuscript that has been accepted for publication. As a service to our customers we are providing this early version of the manuscript. The manuscript will undergo copyediting, typesetting, and review of the resulting proof before it is published in its final citable form. Please note that during the production process errors may be discovered which could affect the content, and all legal disclaimers that apply to the journal pertain.
} 
Conclusions-A novel SG-KS-4D-MRI acquisition method capable of reconstructing rebinning artifact free high resolution 4D-MRI images was used to quantify pancreas tumor motion. The resultant pancreatic tumor motion trajectories agreed well with 2D-cine-MRI and 4D-CT. The pancreatic tumor volumes shown in the different phases for the SG-KS-4D-MRI were statistically significantly more consistent than those in the 4D-CT.

\section{Introduction}

Accuracy of radiation therapy treatment is challenged by ventilation-induced tumor and organ motion, which is especially prominent for lung and upper abdominal tumors. Without precise knowledge of the motion, a generic margin is typically used. Due to individual variations in anatomy and breathing, the generic margins can over- or underestimate the actual motion and lead to dosimetric inaccuracies ${ }^{1,2}$. Therefore, quantification of respiration-induced motion for tumor and critical organs can help clinicians define individualized treatment margins and thus minimize normal tissue doses without compromising tumor dose coverage. Four-dimensional computed tomography (4D-CT) has been widely adopted for lung cancer patients, but is insufficient for abdominal organs due to both resorting artifacts and the poor soft tissue contrast ${ }^{3,4}$. At the same time, use of MRI is particularly attractive for abdominal imaging due to superior soft tissue contrast. Cine twodimensional MRI (2D-MRI) can be oriented to the dominant tumor motion plane for realtime imaging. 5,6

However, unlike lung tumors, abdominal tumors are often surrounded by serial organs. A single 2D plane is likely insufficient to describe the complex motion of these organs. In the radiation therapy community, efforts on four-dimensional magnetic resonance imaging (4DMRI) have been made from multiple groups. Cai J, et al. introduced a 4D-MRI protocol using body area as surrogate to retrospectively resort cine $2 \mathrm{D}-\mathrm{MRI}$ images. ${ }^{7}$ Tryggestad E, et al. developed a longer-duration MRI and post-processing technique based on cine 2DMRI images to derive the average or most-probable state of mobile anatomy. ${ }^{8} \mathrm{Hu} \mathrm{Y}$, et al. used a navigator triggered image acquisition at preselected respiratory amplitudes for 4DMRI, which was also based on 2D acquisition. ${ }^{9}$ Stemkens B, et al compared 2 surrogate signals (external bellows and internal navigator) and 2 MR sampling strategies (Cartesian and radial) and constructed 4D-MRI by resorting 2D K-space data. ${ }^{10}$ All of these 4D-MRI methods have adopted similar concept used in 4D-CT and have shown promise in capturing more comprehensive anatomical information, however they have also inherited the same stitching artifacts resulted from 2D imaging-space resorting ${ }^{7-11}$. Another significant limitation is the anisotropic resolution due to the large slice thickness of cine 2D-MRI.

To overcome these limitations, we have recently demonstrated the feasibility of a SGKS-4D-MRI technique with 3D radial sampling and self-gating based $\mathbf{K}$-space sorting to provide respiratory phase resolved 3-dimensional (3D) MRI images. ${ }^{12}$ The SG-KS-4D-MRI technique has several potential advantages over existing 4D-MRI methods, including an isotropic high spatial resolution of $1.56 \mathrm{~mm}$ with a fixed scan time of 8 minutes and minimal intra-phase motion artifacts. Demonstration of this novel 4D-MRI technique has been performed in phantom, healthy volunteers and two liver patients and geometric accuracy of the method has been studied. 
The purpose of this study is to present our early clinical experience in assessing the effectiveness of SG-KS-4D-MRI to evaluate pancreatic tumor motion, where 4D-CT has typically been inadequate. We compare the tumor motion trajectories measured from SGKS-4D-MRI to those measured from cine 2D-MRI images in sagittal and coronal planes, and from $4 \mathrm{D}-\mathrm{CT}$.

\section{Methods and Materials}

\section{Patients}

This study included ten patients ( 5 male and 5 female) with histologically confirmed locally advanced or boarderline resectable pancreatic cancer. Tumors were located in the head and body of the pancreas in 5 and 4 patients, respectively, with one patient presenting with synchronously distinct tumors in the head and tail of pancreas. Gross tumor volumes (GTVs) ranged from $25-103 \mathrm{cc}$ with an average volume of $51 \mathrm{cc}$. Additional patientspecific variables are noted in Table 1.

\section{Imaging study}

All patients underwent $\mathrm{CT}$ and MRI imaging studies under a prospective protocol approved by the institutional review board (IRB). The time interval between CT and MRI studies was less than 1 week. Patients were in a head-first supine position with arms up. CT scans were performed on a 16-slice scanner (Optima CT580 ${ }^{\mathrm{TM}}$; GE Healthcare, Milwaukee, WI) equipped with the Real-time Position Management $\left(\mathrm{RPM}^{\mathrm{TM}}\right)$ system (Varian Medical Systems, Palo Alto, CA) and AdvantageSim ${ }^{\mathrm{TM}}$ 4D software (GE Healthcare, Milwaukee, WI). 4D-CT scans were performed in cine mode with the following parameters: $120 \mathrm{kV}$, variable $\mathrm{mA}$, gantry rotation period of 1 second, and slice thickness of $2.5 \mathrm{~mm}$. The cine duration time was set to be the patient's breathing period plus 1.5 seconds.

MRI scans were performed on a 3.0 Tesla system (MAGNETOM Verio ${ }^{\mathrm{TM}}$, Siemens Healthcare, Germany) equipped with a 6-channel body matrix coil. No special immobilization was used. The imaging protocol included an 8-minute free-breathing SGKS-4D-MRI scan (prescribed isotropic spatial resolution $=1.56 \mathrm{~mm}$, a cubic imaging volume of $300 \times 300 \times 300 \mathrm{~mm}^{3}$, flip angle $=10^{\circ}$, TR/echo time $(\mathrm{TE})=5.8 / 2.6 \mathrm{~ms}$, readout band width $=399 \mathrm{~Hz} /$ pixel) and two 1.2 minute free-breathing cine 2D-MRI spoiled gradient recalled echo (GRE) scans with orthogonal imaging planes traversing the tumor (351 ms/ frame, 1.56-mm in-plane spatial resolution, 8-mm slice thickness).

\section{D-MRI sequence design, data sorting and image reconstruction}

The SG-KS-4D-MRI method has been described. ${ }^{12}$ In short, a spoiled GRE sequence with 3D radial-sampling K-space trajectory and one-dimensional (1D) projection-based SG has been implemented for image acquisition. The $k$-space is filled using $2 \mathrm{D}$ golden means ordering. ${ }^{13}$ To achieve a reasonable scan time, a total of 73,005 imaging lines are acquired in 8 minutes. Superior-inferior (SI) SG lines are inserted at every 15 imaging lines or an interval of approximately $98 \mathrm{~ms}$ to serve as a respiratory motion surrogate for the 15 lines that follow. Before retrospective $k$-space resorting, the data acquired during the respiratory cycles with abnormal durations or outlier end-expiratory locations (i.e. defined as mean \pm 2 
standard deviations) are discarded. The remaining data are then resorted into one of 10 respiratory phases according to their associated motion surrogate. Approximately 6000 7,000 lines are available in each respiratory phase for image reconstruction using a conjugate-gradient (CG) SENSE method with self-sensitivity calibration implemented in MATLAB (Mathworks, Natick, MA) and an approximately eight-fold acceleration. The total reconstruction time is about 5 hours using a server configured with 12-core Intel Xeon CPU and 96GB of memory.

\section{Comparison of 4D tumor trajectories}

Tumor trajectories resulting from the SG-KS-4D-MRI were compared to those from singleslice cine 2D-MRI and 4D-CT. Superior-inferior (SI), anterior-posterior (AP) and mediallateral (ML) tumor motion were extracted from the retrospectively sorted SG-KS-4D-MRI and 4D-CT images. For the cine 2D-MRI, we extracted SI and AP motion from the sagittal plane image set, and ML motion from the coronal plane image set (the ML motion is usually small so there is less concern about out-of-plane motion). A template matching method based on maximized cross-correlation was used to track tumor motion trajectories, as introduced in a previous study ${ }^{14,15}$. A template encompassing GTV on the first frame was created. The center of the template was then moved to match to the following target frames of cine 2D-MRI. The total processing time for the 200 frames was about 3 seconds. The match of each following frame to the first referenced frame was verified and manually corrected if abnormal match was observed. Peak detection was applied to the tumor trajectory curve, and irregular breathing cycles with the same definition as that for selfgating lines of SG-KS-4D-MRI were discarded. Each of the remaining breathing cycles was then equally divided to 10 phases, and the average position of each phase from multiple breathing cycles was defined to be the tumor trajectory from cine 2D-MRI. For the clinical $\mathrm{CT}$ session, a contrast CT scan was usually performed. For the research MRI session, a contrast MRI scan was performed with patient holding their breath at exhale. These contrast scans were registered to the corresponding end-of-exhale 4D scan to assist target definition. Tumor volumes were drawn at the end of exhalation phase in the SG-KS-4D-MRI and 4DCT images, and then mapped to the other phases using deformable registration in VelocityAI ${ }^{\mathrm{TM}}$ (Varian, Palo Alto, CA). The mapped contours were evaluated against the image information for each breathing phase, and any differences in the mapped contours were manually corrected. The coordinates of the contours' center of mass were then extracted for tumor motion trajectory comparisons.

The difference $\left(\mathrm{D}_{\text {diff }}\right)$ in average motion amplitudes from each of ten phases derived using the three imaging methods was calculated. The correlation coefficient (CC) between the three different imaging methods was also calculated. Standard deviations of the GTV volumes (GTV_ $\sigma$ ) from ten breathing phases were calculated from both SG-KS-4D-MRI and $4 \mathrm{D}-\mathrm{CT}$. CC is defined using following equations:

$S_{x x}=\sum x^{2}-\frac{\left(\sum x\right)^{2}}{10} ; S_{y y}=\sum y^{2}-\frac{\left(\sum y\right)^{2}}{10} ; S_{x y}=\sum x y-\frac{\left(\sum x\right)\left(\sum y\right)}{10} ; C C=\frac{S_{x y}}{\sqrt{S_{x} S_{y}}} ;$ in which $\mathrm{x}$ is the displacement in imaging modality one, and y is the displacement in imaging modality two, and number 10 represents the ten breathing phases. The Wilcoxon signed rank test was 
used to compare the GTV_ $\sigma$ calculated on the two imaging modalities and a $p$ value $<0.05$ was considered to be statistically significant.

\section{Results}

All patients went through SG-KS-4D-MRI, cine 2D-MRI and 4D-CT scans. Patient \#3 failed 4D-CT due to irregular breathing during scanning. In general, this novel SG-KS-4DMRI sequence produced phase-resolved 3D-MRI images free of the "stitching" artifacts that one often observes on 4D-CT. Figure 1 shows an example set of patient 4D-MRI vs. 4D-CT scans on a sagittal plane from end of exhalation phase, in which a "stitching" artifact is seen on 4D-CT (not seen on KS-SG-4D-MRI), due to the unavoidable slight mismatches between cine couch positions.

Figure 2 shows an example patient's cine 2D-MRI acquired on sagittal and coronal planes, and the motion trajectories derived from the 200 image frames. Note that the sagittal images and coronal images were not acquired at the same time, resulting in a notable phase shift when comparing the 2 breathing trajectories. Figure 3 shows an example patient's SGKS-4D-MRI and 4D-CT in five selected breathing phases. SG-KS-4D-MRI shows superior soft tissue contrast compared with the 4D-CT, particularly in structures such as the hepatic vasculature. Figure $4(\mathrm{a}-\mathrm{c})$ shows the comparison of the tumor motion trajectories measured from the center of volume/area from SG-KS-4D-MRI, cine 2D-MRI and 4D-CT for an example patient. Figure 4d shows the 3D rendering of the GTV for selected phases, illustrating the out-of-plane motion of the target volume (one aspect of motion which cannot be evaluated with cine 2D-MR).

Table 2 summarizes the comparison results. On average, the tumor motion trajectories matched well between SG-KS-4D-MRI and cine 2D-MRI in SI direction with a mean CC of $0.93 \pm 0.03$. The motion trajectories matched reasonably well between SG-KS-4D-MRI and cine 2D-MRI in AP and ML directions with mean CC of $0.83 \pm 0.10$ and $0.74 \pm 0.18$ respectively. The average absolute $\mathrm{D}_{\text {diff }}$ comparing SG-KS-4D-MRI and cine 2D-MRI was $0.83 \pm 0.52 \mathrm{~mm}, 0.40 \pm 0.17 \mathrm{~mm}$ and $0.48 \pm 0.24 \mathrm{~mm}$ for SI, AP and ML directions respectively. Good correlation between SG-KS-4D-MRI and 4D-CT was observed in SI direction with an average $\mathrm{CC}$ of $0.91 \pm 0.06$, and a fair correlation in AP direction with an average CC of $0.72 \pm 0.16$. The motion trajectories from SG-KS-4D-MRI and 4D-CT were only poorly correlated in the ML direction with an average CC of $0.44 \pm 0.24$, as one might expect when the ML motions are generally small. The average absolute $\mathrm{D}_{\text {diff }}$ comparing SGKS-4D-MRI and 4D-CT were $1.09 \pm 0.43 \mathrm{~mm}, 0.53 \pm 0.24 \mathrm{~mm}$ and $0.47 \pm 0.26 \mathrm{~mm}$ for SI, AP and ML directions, respectively. The average GTV_ $\sigma$ calculated from ten breathing phases was $0.81 \mathrm{cc}$ and $1.02 \mathrm{cc}$ for SG-KS-4D-MRI and 4D-CT respectively. Wilcoxon signed rank test of GTV_ $\sigma$ from comparison of SG-KS-4D-MRI and 4D-CT resulted in a p value of 0.012 .

\section{Discussion}

Previous work has demonstrated the feasibility of this novel SG-KS-4D-MRI technique in providing respiratory phase-resolved 3D-MRI images with several advantages over 2D slice 
rebinning-based 4D-MRI methods, including isotropic high resolution of $1.56 \mathrm{~mm}$ using a 3D radial acquisition, fixed scan time and absence of artifacts from mismatching 2D slices. ${ }^{12}$ In this study, we report the initial clinical experience of SG-KS-4D-MRI image analysis for pancreatic cancer patients. Tumor trajectories derived from this novel 4D-MRI agreed well with the 3D trajectories derived from sagittal and coronal cine 2D-MRI acquired on the same day, indicating this SG-KS-4D-MRI is capable of measuring tumor respiratory motion at least as well as the limited information available from cine 2D-MRI. We also showed in this study that the SG-KS-4D-MRI trajectories agreed well with those from 4D$\mathrm{CT}$ in SI and AP directions, though less well for the limited motions seen in the ML direction. While 4D-CT can only be used as a reference and not the ground truth due to its aforementioned limitations, the comparison shows that SG-KS-4D-MRI may at least replace 4D-CT for abdominal imaging without radiation.

Several issues contribute to the poor correlations in the ML direction. First, the breathing motion in the ML direction is generally small (range of 1 to $2 \mathrm{~mm}$ ), which is very close to the image resolution, so uncertainties in detection of the motion are of the same size as the actual motion, leading to poor correlation. Second, the two imaging dataset scans were obtained on different days with the possibility of varying body positions and breathing patterns. It has also been reported that $4 \mathrm{D}-\mathrm{CT}$ is prone to error if the breathing pattern is irregular ${ }^{16}$. Finally, due to the unique data acquisition and reconstruction method used in the SG-KS-4D-MRI, the images reflect an average motion, while a 4D-CT frame is "stitched" from multiple axial snapshots based on the same phase stamp. In 4D-CT acquisition, the variation in breathing over the acquisition time could result in binning artifacts. In comparison, observing the breathing motion over a longer period in the SG-KS-4D-MRI may result in more representative motion data than "snapshot" techniques.

The novel SG-KS-4D-MRI sequence makes phase resolved 3D-MRI scans free of "stitching" artifacts, which differentiates this 4D-MRI technique from other existing methods that use sequential slice-by-slice acquisition. Our initial clinical experience with pancreatic cancer patients confirms that this advantage holds not only for phantom, but also for real patients. It should be noted that the accuracy in determination of respiratory phase is critical to artifact-free 4D-MRI images. In our SG-KS-4D-MRI method, 1D projection linebased self-gating signal was used as the surrogate for data rebinning, where the Fourier Transform of the line is essentially a projection of the imaging volume onto the SI axis. The choice of this surrogate is directly based on tumor respiratory motion. The projections that were out of phase determined from these 1D projection lines were discarded before 4D image reconstruction which prevents this novel SG-KS-4D-MRI method from producing phase-induced artifacts. For patients that had irregularities in breathing, a larger percentage of projection lines had to be discarded, which might lead to insufficient data for 4D-MRI image reconstructions, however, the general anatomical structure is still preserved. For example, patient \#3 had an irregular breathing pattern which resulted in a failed 4D-CT, but only a lower than desired signal in the reconstructed 4D-MRI. Implementation of additional coaching and monitoring steps can potentially improve SG-KS-4D-MRI acquisition. For patients with irregular breathing patterns, more projection lines can be acquired with the expense of imaging time for better quality images. One of the advantages of using MRI for $4 \mathrm{D}$ imaging is that it has no radiation dose, so individualized scan time can be used 
depending on each patient's breathing regularity. One possible approach is to divide the 8minute acquisition into two parts. The first 3-minute acquisition will be used for both breathing pattern evaluation and image reconstruction. Depending on the evaluation from the first part, the second 5-minute acquisition can be kept constant or extended.

Another result worth pointing out is the statistically significant improvement of standard deviation of GTVs calculated from ten breathing phases on SG-KS-4D-MRI vs. 4D-CT. Pancreatic tumors are relatively rigid, so we expect a relatively constant tumor volume from one breathing phase to another. A difference in the GTVs from different breathing phases is likely to be a result of deformable registration or manual contouring. Our data showed that this standard deviation is smaller for GTVs derived from SG-KS-4D-MRI than those derived from 4D-CT; indicating a more consistent tumor volume definition from SGKS-4D-MRI. Although the result might be affected by the sample size, it is to our knowledge the first time that the improvement of contouring consistency of 4D-MRI vs. 4DCT has been shown. One explanation for this observation is that the "stitching" artifacts in 4D-CT tends to vary from one frame to another and contributes to greater tumor volume variation. The blurring artifacts in the SG-KS-4D-MRI tend to affect all image frames equally and result in more consistent volume measurement.

Our current SG-KS-4D-MRI images appear to be noisy. The noisy character seen in the images is due to three major factors during the MR acquisition, each with its specific remedy that can be implemented in future 4D-MRI acquisition. First, large gradient jumps in k-space may cause eddy current effects that could potentially degrade image quality. Further development in smoother $\mathrm{k}$-space sampling trajectories is underway to minimize these effects. Second, aggressive under-sampling, especially in patients with irregular breathing patterns, may result in noticeable streaking artifacts, which should be mitigated by simply extending the scan time or adopting advanced reconstruction methods that exploit the spatiotemporal correlation in the multi-channel dataset. Third, off-resonance due to $\mathrm{B}_{0}$ inhomogeneity may contribute to some of the residual noise like artifacts, as the volumetric acquisition required a much larger shimming volume than a 2D protocol, which may degrade the shimming quality. More thorough shimming in the target excitation volume may help alleviate these artifacts by suppressing the signal from anatomic structures far away from the iso-center. It is also possible to improve the signal-to-noise ratio using a state of the art denoising method. ${ }^{17,} 18$ Efforts are needed to further improve the tumor contrast, which can potentially be achieved by introducing T2-weighting to the proposed technique using balanced steady-state free precession acquisition or T2-preparation techniques. ${ }^{9}$ One additional limitation of this pilot study is that only ten patients for one treatment site were imaged. Future work will pursue additional imaging of a wider selection of patients and treatment sites.

\section{Conclusion}

A novel 4D-MRI imaging sequence, SG-KS-4D-MRI, was employed to acquire abdominal images of pancreatic cancer patients. SG-KS-4D-MRI is free of the "stitching" artifacts which are often seen in existing 4D-MRI (which is based on resorted 2D cine MRI) or in 4D-CT scans. Pancreatic motion trajectories described by SG-KS-4D-MRI agreed well with 
those by 4D-CT and 2D sagittal cine MRI in the SI direction. SG-KS-4D-MRI results in substantially improved spatial resolution and voxel isotropy, and gives an average motion pattern that may be more representative of the expected breathing motion than single instance information. SG-KS-4D-MRI also results in statistically more consistent tumor volume definition across ten breathing phases.

\section{Acknowledgments}

This work is supported by NCI grant 1R03CA173273-01

\section{References}

1. Nelson C, Balter P, Morice RC, et al. Evaluation of tumor position and PTV margins using image guidance and respiratory gating. Int J Radiat Oncol Biol Phys. 2010; 76:1578-1585. [PubMed: 20137865]

2. Whitfield G, Jain P, Green M, et al. Quantifying motion for pancreatic radiotherapy margin calculation. Radiother Oncol. 2012; 103:360-366. [PubMed: 22410203]

3. Li G, Caraveo M, Wei J, et al. Rapid estimation of 4DCT motion-artifact severity based on 1D breathing-surrogate periodicity. Med Phys. 2014; 41:111717. [PubMed: 25370631]

4. Watkins WT, Li R, Lewis J, et al. Patient-specific motion artifacts in 4DCT. Med Phys. 2010; 37:2855-2861. [PubMed: 20632597]

5. Akino Y, Oh RJ, Masai N, Shiomi H, Inoue T. Evaluation of potential internal target volume of liver tumors using cine-MRI. Med Phys. 2014; 41:111704. [PubMed: 25370618]

6. Lever FM, Lips IM, Crijns SP, et al. Quantification of esophageal tumor motion on cine-magnetic resonance imaging. Int J Radiat Oncol Biol Phys. 2014; 88:419-424. [PubMed: 24321785]

7. Cai J, Chang Z, Wang Z, Paul Segars W, Yin FF. Four-dimensional magnetic resonance imaging (4D-MRI) using image-based respiratory surrogate: a feasibility study. Med Phys. 2011; 38:63846394. [PubMed: 22149822]

8. Tryggestad E, Flammang A, Han-Oh S, et al. Respiration-based sorting of dynamic MRI to derive representative 4D-MRI for radiotherapy planning. Med Phys. 2013; 40:051909. [PubMed: 23635279]

9. Hu Y, Caruthers SD, Low DA, Parikh PJ, Mutic S. Respiratory amplitude guided 4-dimensional magnetic resonance imaging. Int J Radiat Oncol Biol Phys. 2013; 86:198-204. [PubMed: 23414769]

10. Stemkens B, Tijssen RH, de Senneville BD, et al. Optimizing 4-Dimensional Magnetic Resonance Imaging Data Sampling for Respiratory Motion Analysis of Pancreatic Tumors. Int J Radiat Oncol Biol Phys. 2015

11. Liu Y, Yin FF, Chang Z, et al. Investigation of sagittal image acquisition for 4D-MRI with body area as respiratory surrogate. Med Phys. 2014; 41:101902. [PubMed: 25281954]

12. Deng Z, Pang J, Yang W, et al. Four-dimensional MRI using three-dimensional radial sampling with respiratory self-gating to characterize temporal phase-resolved respiratory motion in the abdomen. Magn Reson Med. 2015

13. Chan RW, Ramsay EA, Cunningham CH, Plewes DB. Temporal stability of adaptive 3D radial MRI using multidimensional golden means. Magn Reson Med. 2009; 61:354-363. [PubMed: 19165897]

14. Shi X, Diwanji T, Mooney KE, et al. Evaluation of template matching for tumor motion management with cine-MR images in lung cancer patients. Med Phys. 2014; 41:052304. [PubMed: 24784397]

15. Cai J, Read PW, Altes TA, Molloy JA, Brookeman JR, Sheng K. Evaluation of the reproducibility of lung motion probability distribution function (PDF) using dynamic MRI. Phys Med Biol. 2007; 52:365-373. [PubMed: 17202620]

16. Persson GF, Nygaard DE, Brink C, et al. Deviations in delineated GTV caused by artefacts in 4DCT. Radiother Oncol. 2010; 96:61-66. [PubMed: 20570002] 
17. Sheng K, Gou S, Wu J, Qi SX. Denoised and texture enhanced MVCT to improve soft tissue conspicuity. Med Phys. 2014; 41:101916. [PubMed: 25281968]

18. Lebrun M. An analysis and implementation of the BM3D image denoising method. Imaging Processing On Line. 2012; 2:175-213. 


\section{Summary}

Cine 2D-MRI is useful when monitoring abdominal tumor motion due to its superior soft tissue contrast. To quantify 3D tumor motion, current 4D-MRI methods are 2D-based, and limited in resolution and by artifacts. In this study, a SG-KS-4D-MRI method based on 3D k-space sampling was implemented for pancreatic tumor motion monitoring. The resultant 4D-MRI images with high isotropic resolution showed fewer artifacts, better soft tissue contrast and more consistent tumor volumes than those of 4D-CT. 

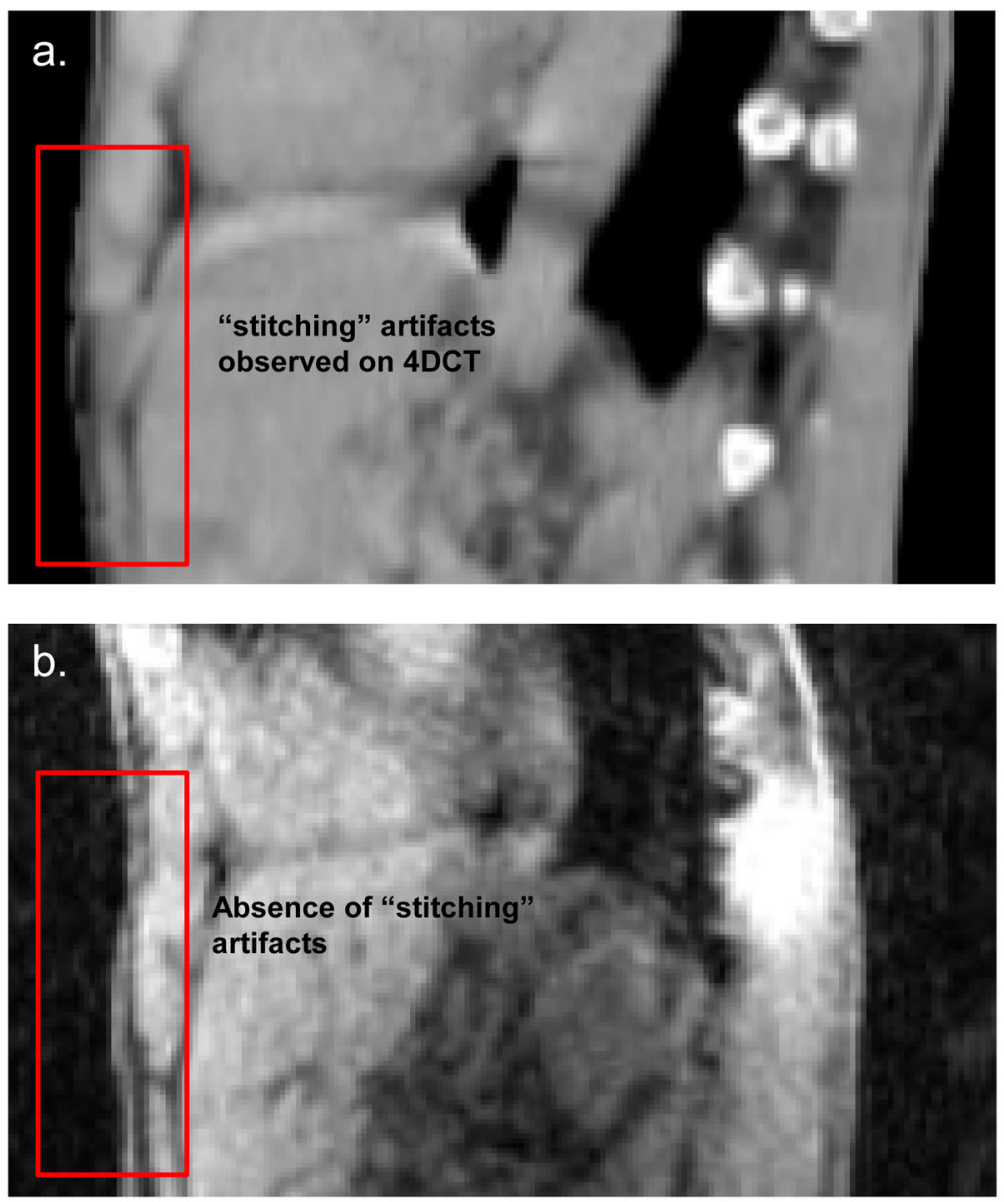

Figure 1.

An example of "stitching" artifact on 4D-CT (a); and absence of the artifact on $S G-K S-4 D$ MRI (b). 

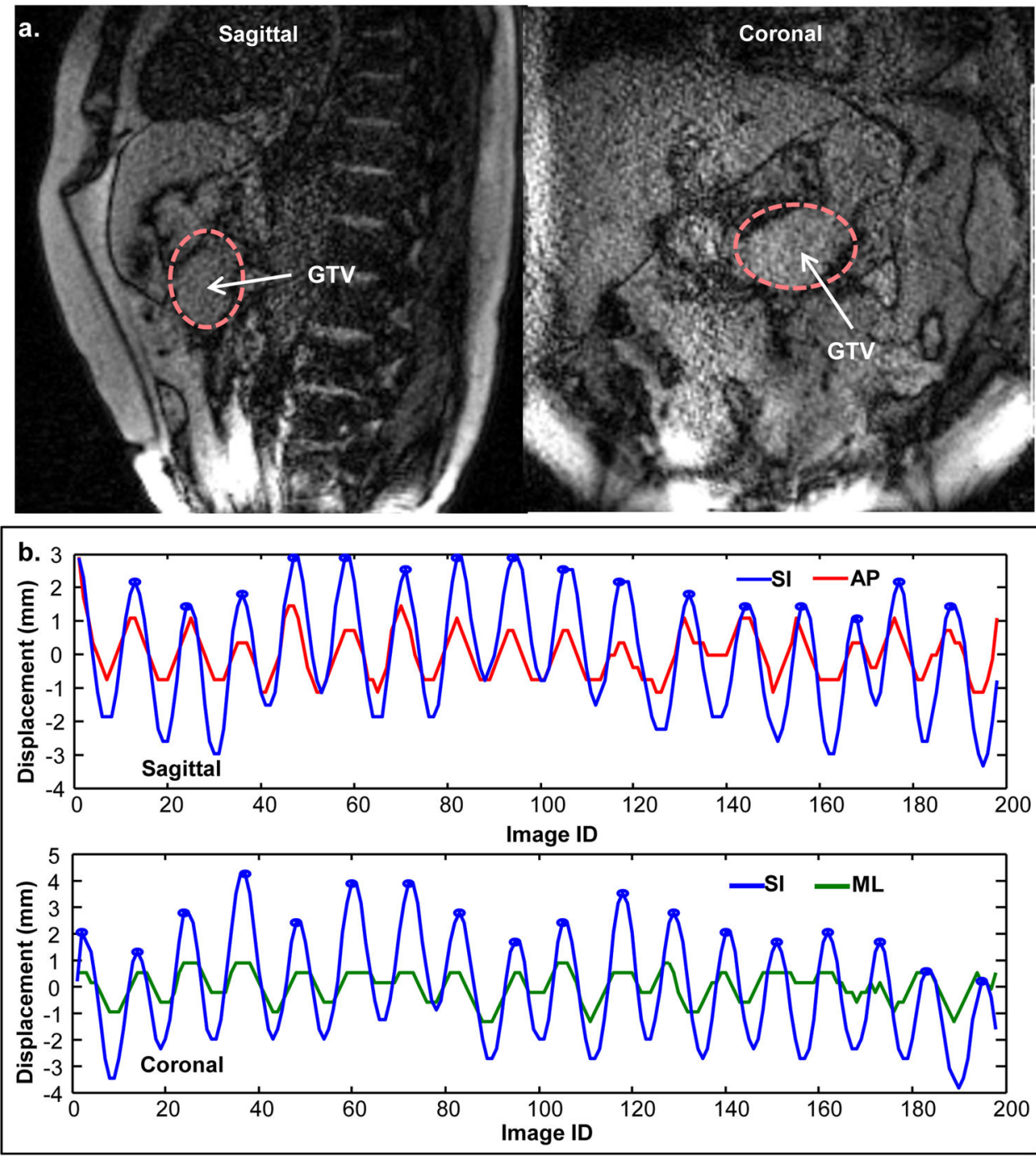

Figure 2.

An example of a patient's 2D cine MRI in sagittal and coronal planes (a); and the respiratory motion trajectories derived from the 2D cine MRI in sagittal and coronal planes. (b) 

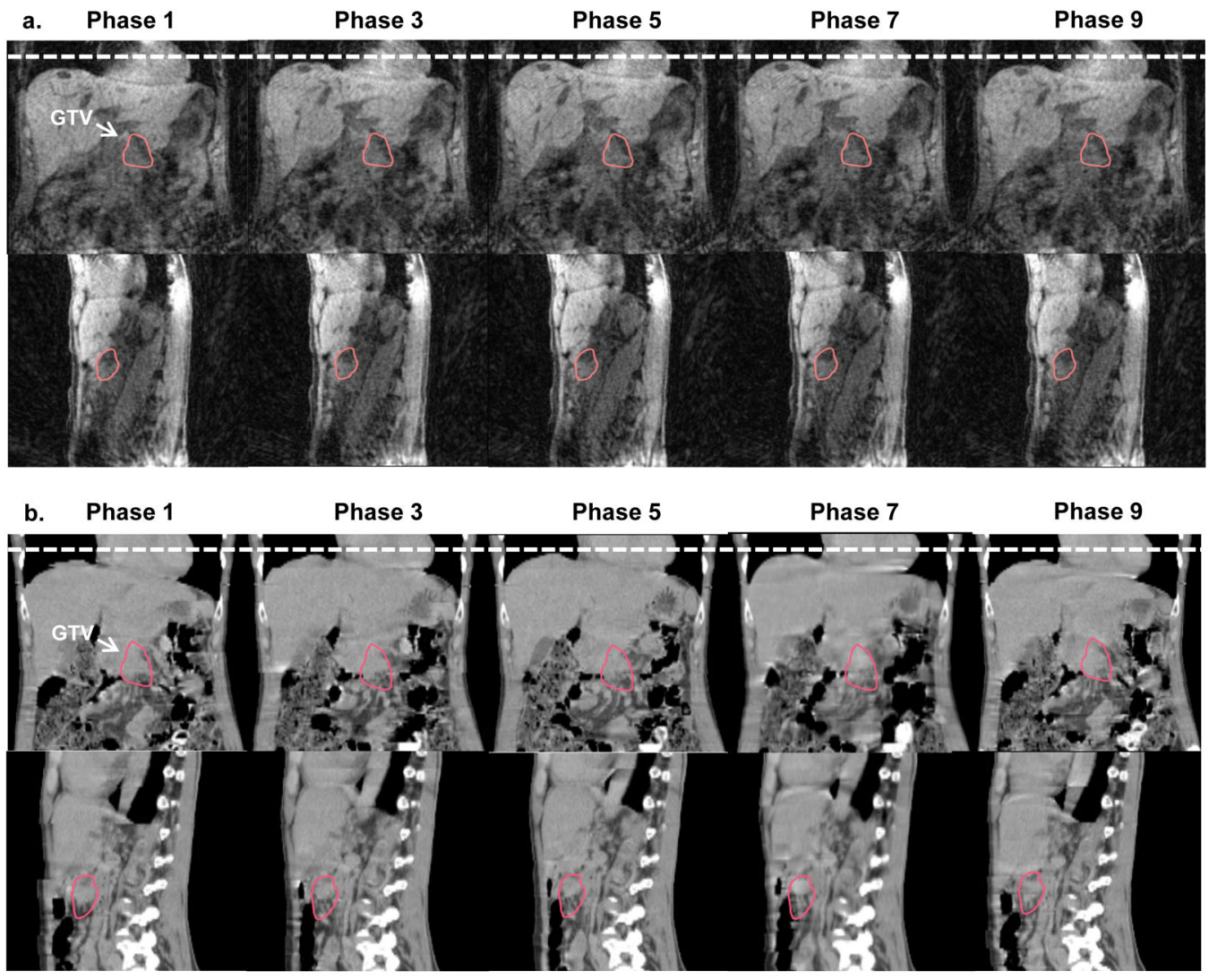

Figure 3.

An example of a patient's 4D-CT (a) and SG-KS-4D-MRI (b) in selected phases, with the GTV shown on the image from each phase. 

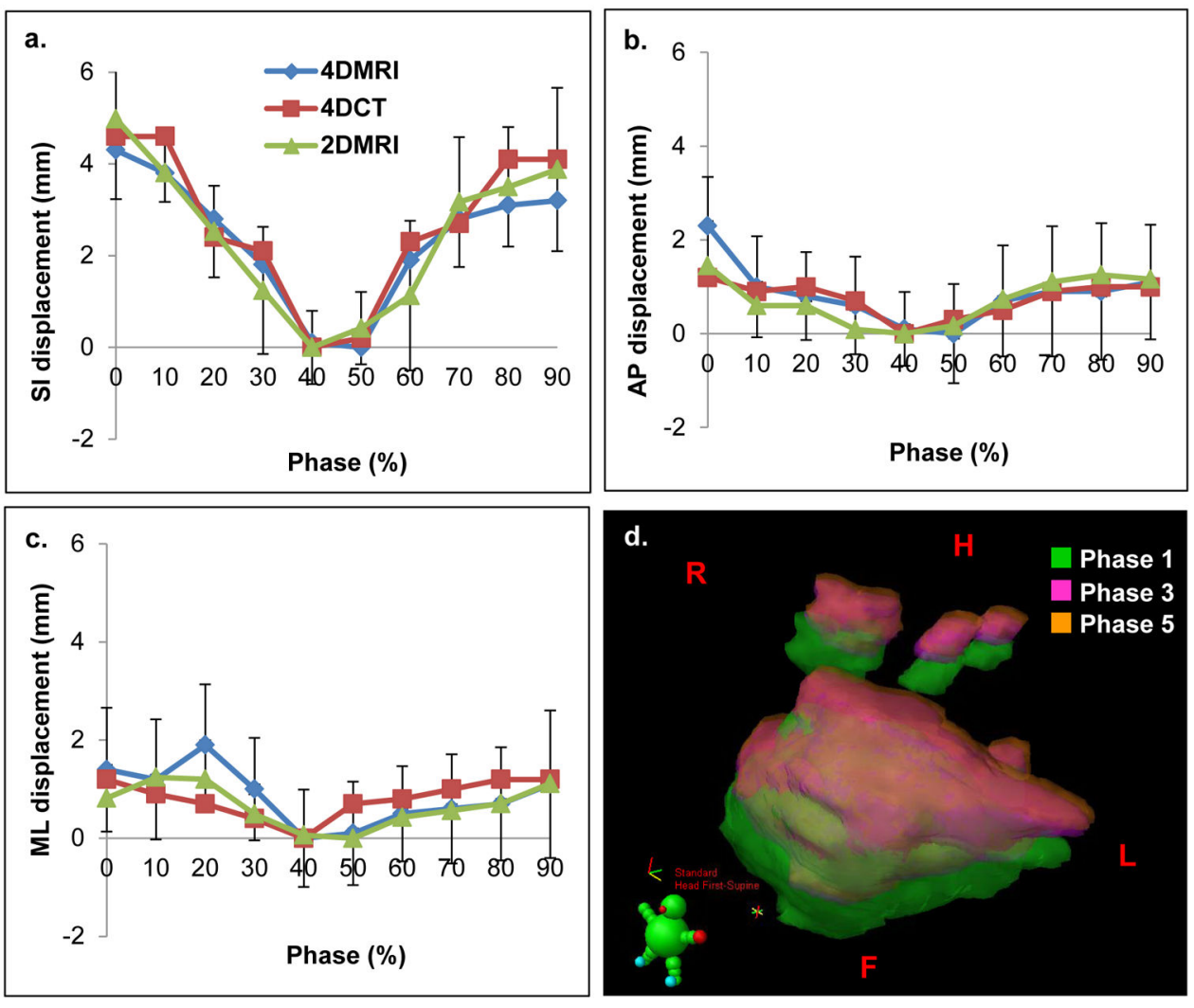

Figure 4.

An example of comparisons of a patient's motion trajectories in SI (a), AP (b) and ML (c) directions, between SG-KS-4D-MRI, 4D-CT and 2D-MRI; the error bars in the plot are the standard deviations of measurements from 2D-MRI with multiple breathing cycles from one minute continuous MRI imaging; a 3D surface rendering of GTVs in three selected phases. (d) 


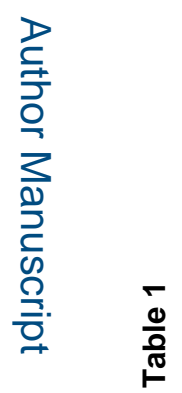

로을

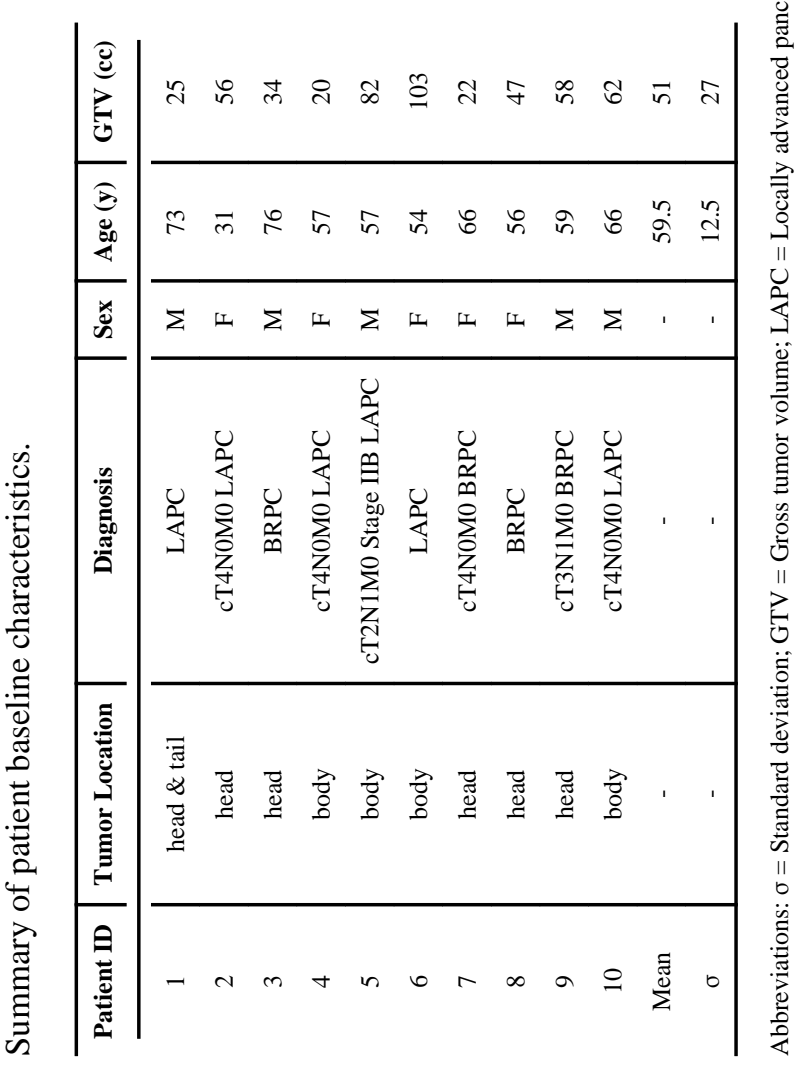

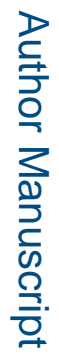


